\title{
Categorización de enfermedades: un estudio sobre expertos y profanos
}

\author{
Asunción López Manjón \\ Universidad Autónoma de Madrid
}

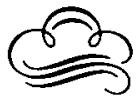

Resumen

Se llevó a cabo un estudio sobre la diferencia entre expertos y profanos respecto a la categorización de las enfermedades utilizando 60 sujetos (20 médicos de familia, 20 enfermeras y 20 profanos universitarios). La similitud percibida de varias enfermedades se ha estudiado presentando una tarea de agrupamiento que incluía 29 enfermedades. El contenido de los agrupamientos se analizó mediante un análisis de conglomerados. No bubo diferencias significativas entre los tres grupos en la clasificación jerárquica excepto en las categorías subásicas. Los médicos establecieron y usaron un mayor número de suclasificaciones que las enfermeras y los profanos. La localización anatómica fue la dimensión más importante utilizada para agrupar las enfermedades en todos los grupos. Las enfermedades infecciosas se agruparon juntas en el caso de los médicos y enfermeras, pero no asi en el de los profanos.

Palabras clave: Cognición enfermedad, expertos-profanos, clasificación de enfermedades.

\section{Abstract}

A study about expert-naive of illness categorization has been carried out using 60 adult subjects (20 family physicians, 20 nurses and 20 lay subjects). Similarity perceived across different illnesses bas been studied presenting a grouping task containing 29 different illnesses. There were no significant differences between the three groups on the hirarchical clasification except in sub-basic categories. The pshysicians establish and use a bigger amount of subclasifications than nurses and lay subjects. The most important dimension for grouping illness was anatomical location across all groups of subjects. Grouping content was analyzed by cluster analysis. The infectious illnesses were grouped by psysicians and nurses, but this wast not the case in lay subjects.

Key words: Illness cognition, expert-naive, illness clasification.

Dirección de la autora: Departamento de Psicología Básica. Universidad Autónoma de Madrid. Campus de Cantoblanco. 28049 Madrid.

Agradecimientos: La investigación que ha dado lugar a este artículo forma parte de la tesis doctoral de la autora dirigida por Mario Carretero. Quiero expresar mi agradecimiento a Mikel Asensio, José Antonio León, Nacho Pozo, Puy Pérez Echeverría, Juan Antonio Huertas y por supuesto a Mario Carretero. 


\section{INTRODUCCION}

En los últimos años y fundamentalmente a partir del artículo The Common Sense Representation of illness Danger (Leventhal, Meyer y Nerez, 1980) se ha puesto de manifiesto la importancia que poseen las teorías implícitas o intuitivas sobre la enfermedad a la hora de interpretar, comprender y regular las conductas hacia la salud (para una revisión véase Bishop, 1991a; López Manjón, 1991). A partir de entonces se han desarrollado un gran número de investigaciones que estudian la estructura de dichas representaciones que según Bishop (1991a) pueden dividirse en dos enfoques. El enfoque de los componentes de la representación y el enfoque de las dimensiones o factores que están implicados en la misma. Los estudios realizados bajo el primer enfoque (Leventhal, Meyer y Nerenz, 1980; Leventhal y Nerenz, 1982; Bishop, Briede, Cavazos, Grotzinger y McMahon, 1987) han detectado los siguientes componentes de la representación sobre la enfermedad: a) identidad: lo que es la enfermedad, es decir, el diagnóstico; b) consecuencias: los efectos a largo y corto plazo de la enfermedad; c) duración: el curso temporal de la enfermedad; d) causa: el factor que lleva al comienzo de la enfermedad y e) curación (Lau y Hartman, 1983; Lau, Bernard y Hartman, 1989). Los estudios llevados a cabo bajo el enfoque de las dimensiones (Jenkins, 1966; Jenkins y Zyzanski, 1968; Turk, Rudy y Salovey, 1986) se basan en el supuesto de que existen ciertas dimensiones cognitivas que caracterizan las representaciones sobre la enfermedad y que pueden analizarse a través de técnicas estadísticas tales como el análisis factorial.

Los métodos y objetivos de los dos enfoques son distintos y de ahí que los resultados obtenidos sean diferentes. Los estudios que examinan los componentes de la representación han usado el análisis del contenido de las descripciones de los sujetos sobre su experiencia con la enfermedad, mientras que el trabajo realizado acerca de las dimensiones utilizan escalas de estimación. Además, los dos enfoques examinan aspectos diferentes pero complementarios del mismo fenómeno. Mientras que el enfoque de los componentes se dirige al contenido específico de las representaciones, el enfoque de las dimensiones examina cómo los sujetos evalúan el contenido.

Sin embargo, un aspecto que no ha sido considerado por los estudios anteriores es cómo las representaciones de enfermedades diferentes se relacionan unas con otras. Bishop (1991b) utilizó una tarea de clasificación de un conjunto de enfermedades con el objetivo de analizar cómo los sujetos perciben las relaciones entre ellas. Aplicando el análisis multidimensional encontró dos dimensiones subyacentes a dicha clasificación: contagio y gravedad. D’Andrade, Quinn, Nerlove y Romney (1972) con una muestra de sujetos norteamericanos también encontraron dichas dimensiones utilizando la misma técnica estadística.

El presente estudio se sitúa en la misma dirección que los dos mencionados previamente. Sin embargo, un aspecto no considerado en ninguna de las investigaciones anteriores ha sido la comparación entre expertos y profanos dentro del área de la Medicina. Los estudios realizados sobre expertos y novatos en áreas de conocimiento específicas resultan muy fructíferos para adentrarnos en el conocimiento de las representaciones (véase por ejemplo Chi, Glaser y Farr, 1988).

En este experimento vamos a utilizar la clasificación de enfermedades para obtener las categorías implícitas que utilizan las personas cuando hacen referencia al dominio de la enfermedad. Además estamos interesados en ver si las dimensiones utilizadas por parte de los sujetos profanos son compartidas por 
los expertos. Vamos a estudiar dichas dimensiones a través de las relaciones que se perciben entre un grupo de enfermedades. La tarea a realizar es una tarea de agrupamiento según los criterios que cada sujeto crea adecuados. Por ello nuestro interés es doble: primero observar qué tipo de agrupamientos realizan los diferentes grupos en función de su conocimiento o cercanía con el modelo médico y, por otro, analizar el tipo de respuesta justificativa. Es decir, las razones explícitas que generan los sujetos sobre los agrupamientos realizados. De esta manera podremos observar si los profanos utilizan categorías propias o comparten categorías con los expertos en medicina.

Algunos autores que han trabajado sobre expertos y novatos en el dominio de la Física (Chi, Glaser y Rees, 1982; Chi, Feltovich y Glaser, 1981) sostienen que una diferencia importante entre los dos grupos reside en el tipo de categorías utilizadas para la clasificación de problemas de física. Estos autores pidieron a un grupo reducido de expertos y de novatos que clasificaran jerárquicamente un grupo de problemas de física. Posteriormente se les sugirió subdividir cada grupo. A continuación se les pidió que volvieran a la clasificación inicial e intentaran agruparlos en categorías más amplias. Las estructuras de árbol obtenidas de las clasificaciones jerárquicas diferían según el grupo. Mientras que los expertos realizaban menor número de categorías en la primera clasificación, pero con una estructura de árbol más definida, los novatos utilizaban pocas categorías que agruparan a las categorías realizadas en primer lugar y menor número de subclasificaciones. Examinando el contenido de cada clasificación se detectó que las categorías subordinadas de los expertos correspondían a las categorías iniciales de los novatos. Nuestro objetivo es estudiar si en el dominio de la Medicina también se producen diferencias en la clasificación jerárquica de las enfermedades entre profanos y expertos.

Otro objetivo de este estudio es ver si las dimensiones utilizadas se agotan en la primera clasificación o si el sujeto tiene acceso a otras formas de clasificación. De esta manera también podemos observar si los médicos, al pedirles que clasifiquen las enfermedades de otra manera, se acercan más a los criterios de los profanos o por el contrario disponen de categorías suplementarias del modelo médico. Por último mediante una prueba de recuerdo de la primera clasificación que realizan, queremos comprobar si las categorías y los agrupamientos utilizados son debidos al azar o si están basados en características salientes que pueden ser recordadas sin ningún tipo de error. Los errores que se produzcan pueden ser considerados como el resultado de una clasificación difusa o poco estable. También pensamos que el recuerdo en los expertos será más exacto que el recuerdo en los profanos porque sus categorizaciones son más estables y elaboradas.

Por otro lado, la distinción entre expertos y novatos nos parece un tanto dicotómica. Por tanto, hemos introducido un tercer grupo con una experiencia intermedia, como son el personal de enfermería cuya formación, como es sabido, es más breve y superficial que la de los médicos y más orientada a la práctica. La edad puede ser un factor importante debido a que la experiencia con la enfermedad suele ser mayor a medida que avanza la edad. Helman (1978) encontró diferencias entre grupos diferentes de edad, los más jóvenes compartían modelos más cercanos al modelo médico que las personas de más edad. 


\section{METODO}

\section{Sujetos}

Se utilizaron 20 médicos de familia de diferentes Centros de Salud pertenecientes al INSALUD de Madrid (7 hombres y 13 mujeres), 20 enfermeros del hospital Ramón y Cajal (18 mujeres y 2 hombres) y 20 profanos (18 mujeres y 2 hombres) con estudios universitarios no relacionados con la medicina pertenecientes al personal administrativo y de biblioteca de la Universidad Autóno. ma de Madrid. Todos ellos estaban comprendidos entre los 30 y 40 años (X profanos $\left.=34,15 ; X_{\text {enfermeras }}=34,55 ; X_{\text {médicos de familia }}=31\right)$. No se consideró relevante la variable sexo, ya que en otros estudios (Baumann, Cameron, Zimmerman y Leventhal, 1989) no se han encontrado diferencias entre las estimaciones de las enfermedades entre hombres y mujeres, ni interactúan de manera significativa con otras variables.

\section{Material}

El material consistió en la presentación de tarjetas con los nombres de 29 enfermedades: infarto de miocardio, gripe, fractura de cadera, varicela, SIDA, hepatitis, malaria, polio, neumonia, hipertensión, anginas, artritis, cáncer de pulmón, angina de pecho, catarro, apendicitis, cándes de estómago, jaqueca, diabetes, epilepsia, úlcera, gota, cólico nefrítico, urticaria, acné, miopia, depresión, anemia y sordera. Parte de ellas fueron utilizadas en la investigación de Bishop (1991b). Este autor utilizó 22 enfermedades. De éstas se suprimieron algunas que en un primer estudio piloto resultó que no eran suficientemente conocidas y entre otras decidimos utilizar el nombre por el que se las conoce vulgarmente. También se añadieron otras para que pudieran cubrir la mayor parte de la clasificación médica por especialidades. Las enfermedades cubrían todos los criterios que pudimos imaginar relevantes como infecciosas, no infecciosas, graves-leves, enfermedades infantiles-edad madura-vejez, tratamiento médicoquirúrgico, tratamiento urgente-no urgente, crónicas-agudas, afectación importante vida cotidiana-no afectación, hereditarias-estilo de vida, etc. El nombre de cada enfermedad se presentaba en el centro de una tarjeta blanca de 7,5 × 12,6 $\mathrm{cm}$.

\section{Procedimiento}

Se les presentaba a los sujetos individualmente las correspondientes 29 tarjetas diciéndoles que el objetivo de esta investigación era estudiar cómo las personas categorizan las enfermedades. Se les indicaba que las tarjetas contenían nombres de diferentes problemas relacionados con la salud. Su tarea consistía en agrupar las diferentes tarjetas según la similitud percibida. Podían realizar los grupos que desearan y dejar tarjetas sueltas si consideraban que no existía ninguna relación con las demás. A continuación los sujetos debían realizar las siguientes tareas de clasificación.

\section{1. ${ }^{\mathrm{a}}$ Fase: $10^{\mathrm{a}}$ clasificación}

1.1. Cuando los sujetos habían agrupado las tarjetas, se les pedía el criterio que habían utilizado. Si habían dejado aisladas algunas tarjetas se les pedía que pensaran qué otra enfermedad, aunque no estuviera en las tarjetas, podría agru- 
parse con dicha tarjeta para poder denominar de alguna manera al grupo. A este primer agrupamiento se le denominará categorias básicas.

1.2. La siguiente tarea consistía en que a partir de los agrupamientos realizados (se dejaban encima de la mesa), pensaran si existía alguna posibilidad de subclasificación y que de igual manera mencionaran los criterios que habían utilizado. A esta subclasificación se le denominará categorías subásicas.

1.3. El siguiente paso consistió en volver a la clasificación básica (se volvían a amontonar las tarjetas según la primera clasificación) y perdirles que agruparan los montones que ya habían formado en otros mayores, si existía alguna semejanza entre ellos. También les pedimos que nombraran los criterios utilizados. A estas nuevas categorías construidas se les denominó categorías suprabásicas.

\section{Fase: Reorganización}

Una vez finalizada la fase anterior se mezclaban las 29 tarjetas y se les pedía que las agruparan de otra manera, es decir, si existía otro modo de organizar el conjunto de enfermedades, solicitando los criterios empleados en el agrupamiento. A esta segunda clasificación se le denominará reorganización.

\section{3. ${ }^{\mathrm{a}}$ Fase: Recuerdo de la primera clasificación}

Finalmente se les pedía que volvieran a agruparlas según la primera clasificación que habían realizado (únicamente las categorías básicas), denominándola recuerdo de la primera clasificación. En el caso de que a los sujetos no se les ocurriera agruparlas de otra manera, se dejaba transcurrir un tiempo mientras se charlaba de otros temas, antes de pedirles el recuerdo de la primera clasificación, para que las condiciones no variaran respecto a los sujetos que sí reorganizaban las enfermedades.

El lugar de realización de la prueba fue en los lugares de trabajo correspondientes. La prueba duró aproximadamente entre 20 y 40 minutos.

\section{RESULTADOS}

Se han utilizado tres tipos de análisis. Por una parte el análisis cuantitativo de las categorizaciones realizadas, por otra parte un análisis de los criterios empleados en la justificación de los grupos y, por último, un análisis cualitativo del contenido de los agrupamientos, mediante la técnica de análisis de conglomerados (clusters) realizados por cada grupo.

\section{Análisis cuantitativo de la clasificación}

Para analizar las diferencias cuantitativas entre los tres grupos hemos utilizado las siguientes variables:

1. NCB: número de agrupamientos correspondientes a las categorías básicas.

2. NCSP: número de agrupamientos correspondientes a las categorías suprabásicas.

3. NCSB: número de agrupamientos correspondientes a las categorías subásicas.

4. C70: número de agrupamientos que cubren la mayoría de las 29 enfermedades, el 70 por ciento de las enfermedades, en las categorías básicas. Esta 
variable la hemos utilizado para analizar si ante un mismo número de agrupamientos varía el número de enfermedades que se incluyen dentro de cada grupo.

5. MCBSP: media de los agrupamientos de las categorías básicas que se incluyen en las suprabásicas. Tanto esta variable como la siguiente es una forma de medir el tipo de categorización jerárquica realizada, junto con las variables NCSP y NCSB.

6. MCSBB: media de los agrupamientos de las categorías subásicas que afectan a las categorías básicas.

7: R: número de agrupamientos correspondientes a la reorganización.

8. C70R: número de agrupamientos que cubren el 70 por ciento de la clasificación en la tarea de reorganización.

Utilizando la prueba de Kruskal-Wallis hemos encontrado que no existen diferencias significativas entre los tres grupos en la variable $\mathrm{NCB}\left(\mathrm{X}^{2}=0.4123\right.$; $\mathrm{p}=0.8137), \operatorname{NCSP}\left(\mathrm{X}^{2}=0.4158 ; \mathrm{p}=0.812\right), \mathrm{C} 70\left(\mathrm{X}^{2}=0.438 ; \mathrm{p}=0.803\right)$, $\operatorname{MCBSP}\left(\mathrm{X}^{2}=0.7364 ; \mathrm{p}=0.692\right), \operatorname{MCSBB}\left(\mathrm{X}^{2}=4,168 ; \mathrm{p}=0,1244\right), \mathrm{R}$ $\left(\mathrm{X}^{2}=4,2625 ; \mathrm{p}=0,1187\right)$ y $\mathrm{C} 70 \mathrm{R}\left(\mathrm{X}^{2}=3,9216 ; \mathrm{p}=0,14\right)$. En la Tabla I podemos observar las medias de cada variable obtenidas en cada grupo.

TABLA I

Medias obtenidas por cada grupo en cada variable dependiente

\begin{tabular}{|lccc|}
\hline & PROFANOS & ENFERMERAS & MEDICOS \\
\hline NCSP & 1,2 & 1,15 & 1,4 \\
\hline NCB & 10,55 & 10,45 & 11,1 \\
\hline NCSB & 9 & 11,5 & 15,15 \\
\hline C70 & 5,3 & 4,95 & 5,2 \\
\hline C70R & 3,9 & 2,65 & 4 \\
\hline MCBSP & 16,85 & 18,65 & 15,65 \\
\hline MCSBB & 23,65 & 28,7 & 24,65 \\
\hline R & 8,2 & 5,4 & 8,6 \\
\hline
\end{tabular}

Unicamente existen diferencias significativas en la variable NCSB $\left(\mathrm{X}^{2}=14,174 ; \mathrm{p}=0,0008\right)$. Aplicando la prueba de Scheffe para comparaciones múltiples no paramétricas (San Martín y Pardo, 1989) encontramos que no hay diferencias significativas entre el grupo de profanos $(\bar{X}=9)$ y enfermeras $(\bar{X}=11,5)$, ni entre el grupo de enfermeras $(\bar{X}=11,5)$ y de médicos $(\bar{X}=15,15)$, pero sin embargo, sí existen diferencias entre profanos $(\bar{X}=9)$ y médicos $\overline{(\mathrm{X}}=15,15)$. 
Comparaciones entre la primera clasificación y la reorganización: Utilizando la prueba de Wilcoxon hemos encontrado que existen diferencias significativas $(\mathrm{W}=40,5 ; \mathrm{p}=0,025)$ en el grupo profanos entre el número de agrupamientos utilizados en la primera clasificación $(\bar{X}=10.55)$ y en la reorganización $(\bar{X}=8.2)$ al igual que en el grupo de enfermeras $(\mathrm{W}=23,5 ; \mathrm{p}<0.001)$ en la primera clasificación $(\bar{X}=10,45)$ y reorganización $(\bar{X}=5,4)$. Es decir, se genera un mayor número de agrupamientos en la primera clasificación que en la tarea de reorganización en el grupo de profanos y en el de enfermeras. Esto puede interpretarse como una tendencia a utilizar agrupamientos que incluyan mayor número de enfermedades en la tarea de reorganización que en la primera clasificación (categorías básicas). Más adelante tendremos ocasión de comprobar si los criterios por los que los agrupan son los mismos o generan criterios nuevos en la tarea de reorganización. Con respecto al grupo de los médicos, en estas dos variables no existen diferencias significativas $(W=53 ; p<0.05)$. Los médicos, por tanto generan un número similar de agrupamientos tanto en la primera clasificación como en la de reorganización.

Otra de las comparaciones que nos parece interesante es el número de agrupamientos que cubren el 70 por ciento de la primera clasificación comparándolo con el número de agrupamientos que cubren el 70 por ciento de la reorganización. En el grupo de los profanos, hay diferencias significativas $(\mathrm{W}=32 ; \mathrm{p}<0.05)$ entre $\mathrm{C} 70$ y $\mathrm{C} 70 \mathrm{R}$. Se aprecia un mayor número de agrupamientos que cubren el 70 por ciento de la clasificación en la primera clasificación (categorías básicas) $(\bar{X}=5,3)$ que en la tarea de reorganización $(\bar{X}=3,9)$. En el grupo de las enfermeras, tambié aparecen diferencias $(W=20 ; p<0.005)$ entre la variable $\mathrm{C} 70(\overline{\mathrm{X}}=4,95)$ y $\mathrm{C} 70 \mathrm{R}(\overline{\mathrm{X}}=3,9)$. En el grupo de médicos, también aparecen diferencias significativas $(\mathrm{W}=34 ; \mathrm{p}<0.005)$. Hay un mayor número de agrupamientos que cubren el 70 por ciento de la clasificación en la primera clasificación $(\bar{X}=5,2)$ que en la tarea de reorganización $(\bar{X}=4)$. Parece ser que aunque el número de agrupamientos no se ha reducido en el grupo de médicos entre la primera clasificación y la reorganización, ha cambiado el número de enfermedades que incluyen en cada grupo. En la tarea de reorganización hay una media de 4 grupos que cubren el 70 por ciento de la clasificación. Es decir, aproximadamente 20 enfermedades se sitúan en dicha tarea en 4 grupos, mientras que 20 enfermedades se sitúan en 5,2 grupos en la primera clasificación.

Recuerdo de la primera clasificación: Para evaluar el recuerdo de la primera clasificación (categorías básicas) contabilizamos el número de personas que habían recordado exactamente todas las enfermedades en su grupo correspondiente. Aplicando la prueba de Chi cuadrado obtuvimos diferencias significativas en la distribución de los tres grupos $\left(X^{2}=16,25\right.$; g.l. $\left.=2 ; p=0,0003\right)$. Hay 15 médicos que recuerdan con exactitud la primera clasificación, en comparación con 3 enfermeras y 6 profanos. Además hemos contabilizado el número de enfermedades que cada sujeto no sitúa en el mismo grupo que en la primera clasificación (categorías básicas). Mediante la utilización de la prueba de Anova de un factor (grupo de sujetos), hemos encontrado diferencias significativas $(F=7,083$; $\mathrm{p}=0,0018)$ entre el grupo de profanos $(\overline{\mathrm{X}}=3,3)$ y el grupo de médicos $\overline{\mathrm{X}}=0,45)$, y entre el grupo de médicos y de enfermeras $(\overline{\mathrm{X}}=2,7)$. Sin embargo, no existen diferencias entre el grupo de profanos $(\bar{X}=3,3)$ y el de enfermeras $(\bar{X}=2,7)$. 
2. Análisis de los criterios explícitos utilizados por los sujetos para los agrupamientos

Los datos comentados anteriormente indican diferencias cuantitativas entre los tres grupos. Sin embargo, no han revelado el contenido de dichos agrupamientos. Para analizar dicho contenido además de utilizar el análisis de conglomerados que comentaremos en el siguiente apartado, nos ha parecido conveniente analizar los criterios generados por los propios sujetos para agrupar las diferentes enfermedades. Para ello se ha realizado la siguiente clasificación de los diferentes criterios utilizados por los sujetos:

Criterio 1. Localización: En este criterio se incluyen todas las respuestas justificativas que hacen referencia a la agrupación de las enfermedades por sistemas u órganos afectados. Es decir, las enfermedades se agrupan porque están localizadas en un mismo sistema, afectan a un mismo órgano o son tratadas por el mismo especialista, p. e., enfermedades de corazón, de huesos.

Criterio 2. Causa: Las enfermedades se agrupan por suponer que provienen de una causa común. Por ejemplo, se agrupan porque son infecciosas, hereditarias, debidas al estilo de vida o al estrés.

Criterio 3. Consecuencias: Esta categorización obedece a las repercusiones que se supone la enfermedad causará. Se tiene en cuenta, por tanto, si las enfermedades son graves, leves, afectan a la vida cotidiana o dejan secuelas.

Criterio 4. Tratamiento: Este criterio hace referencia a que diversas enfermedades se ponen juntas porque se curan de manera parecida, e incluso aquí también se han incluido criterios como la prevención, la existencia de enfermedades que pueden prevenirse a través de vacunas. Por ejemplo, la anemia, la úlcera, la diabetes hipertensión se agrupan porque se pueden tratar mediante la dieta.

Criterio 5. Etiquetaje: Hace referencia a si un problema se le considera una enfermedad, un síntoma o un factor de predisposición. Por ejemplo, miopía y sordera no se las considera una enfermedad, mientras que el cáncer de pulmóbn o el de estómago se sitúan juntas porque las dos son cánceres.

Criterio 6. Frecuencia: Se agrupan las enfermedades porque son comunes, frecuentes o raras, p. e., catarro, gripe y jaqueca se agrupan porque son enfermedades comunes.

Criterio 7. Tipo de persona o edades afectadas: Hace referencia al tipo de persona más susceptible de tener esa enfermedad o en qué edades se presenta de forma más característica, p. e., la varicela, polio y anginas se agrupan porque son enfermedades infantiles.

Criterio 8. Tipo de países en que se suelen dar más a menudo, p. e., infarto de miocardio, cáncer de estómago y cáncer de pulmón se agrupan porque son enfermedades de países desarrollados, mientras que la malaria es característica de los países subdesarrollados.

Criterio 9. Síntomas. Si el agrupamiento se realiza porque tienen síntomas parecidos, o por las características de los síntomas como el dolor. p. e., el cólico nefrítico, la úlcera y la apendicitis se agrupan porque todas son enfermedades dolorosas.

Criterio 10. Duración: Si son enfermedades agudas o crónicas. Es decir, hacen referencia al tipo de curso que sigue la enfermedad. Por ejemplo, la hipertensión, la diabetes y epilepsia se agrupan porque son enfermedades crónicas.

Criterio 11. Relación específica: Relación que se establece entre las enfer- 
medades incluidas en un grupo, como por ejemplo, que una es un factor de predisposición de otra, o una lleva a la otra. Por ejemplo, la diabetes puede ser un factor de riesgo de las enfermedades cardiovasculares.

Criterio 12. Mezcla de criterios: Se considera cuando los sujetos suelen suministrar más de un criterio para su agrupamiento.

Criterio 13. Otros: En esta categoría se han incluido aquellos criterios que eran incalificables como «no conoce la enfermedad», «puede ser patológica o no» o afirmaciones puramente anecdóticas.

La justificación de cada agrupamiento generado por cada sujeto se incluyó dentro de cada criterio. De ahí que, por ejemplo, un sujeto pudiera realizar tres grupos según el criterio 1 (Localización: enfermedades de huesos, piel, cardiovasculares), dos grupos según el criterio 2 (Causa: enfermedades causadas por la alimentación y víricas) y dos grupos según el criterio 6 (Frecuencia: comunes e infrecuentes). A partir de dichas puntuaciones se obtuvo la proporción de utilización de cada criterio sobre el número total de agrupamientos para cada sujeto. Esto se realizó acerca de los agrupamientos realizados en la primera clasificación con las categorías básicas, con las categorías suprabásicas y con las subásicas. Al mismo tiempo también se utilizó con las categorías de la prueba de reorganización.

\section{Categorías básicas}

Se realizó un ANOVA $3 \times 13$, entre los tres grupos (profanos, enfermeras y médicos) y para cada uno de los 13 criterios. Los resultados obtenidos muestran que existen efecto principal de la variable criterio $(F=45,649$; g.l. $=12$; $p=1 . O E-4)$ y no existe efecto principal de la variable $G$ rupo $(F=0,822 ;$ g.l. $=2$; $\mathrm{p}=0,5)$. También se observan efectos de interacción entre las dos variables $(\mathrm{F}=2,353 ;$ g.1. $=24 ; \mathrm{p}=0,0003)$.

\section{TABLA II}

Proporciones de utilización de los diferentes criterios para cada grupo, en las categorias básicas de la primera clasificación

\begin{tabular}{lccccccccccccc}
\hline & C1 & C2 & C3 & C4 & C5 & C6 & C7 & C8 & C9 & C10 & C11 & C12 & C13 \\
\hline PROFANOS & .37 & .21 & .14 & .01 & .03 & .01 & .03 & .03 & .05 & .00 & .01 & .08 & .01 \\
\hline ENFERMERAS & .34 & .18 & .09 & .06 & .02 & .00 & .02 & .00 & .07 & .02 & .07 & .11 & .00 \\
\hline MEDICOS & .56 & .14 & .02 & .01 & .05 & .00 & .02 & .00 & .01 & .05 & .06 & .05 & .00 \\
\hline
\end{tabular}

Aunque se ha obtenido efecto principal de la variable Criterio, lo que más nos interesa es el efecto de interacción, es decir, las comparaciones entre las casillas para ver las diferencias en el comportamiento de los tres grupos con respecto a los diferentes criterios. Por tanto, para ver entre qué combinación de variables se establecen las diferencias significativas se realizó la prueba de Tukey (San Martín y Pardo, 1989) (Diferencia mínima significativa = 0,154).

Si comparamos los tres grupos en relación con la utilización de cada criterio podemos observar que la única diferencia significativa se establece en el criterio localización. Los médicos utilizan este criterio con más frecuencia que los profanos y las enfermeras. Estos dos grupos se comportan de manera similar. 
En los demás criterios no ha habido diferencias significativas entre los tres grupos, por tanto, hay una utilización similar de los demás excepto del criterio de localización. Cuando realizamos la comparación intrasujetos, se observan diferencias entre la utilización del criterio localización y los demás criterios en el grupo de los profanos, enfermeras y los médicos. Existe un empleo del criterio localización en mayor medida que demás criterios donde no se establecen diferencias significativas entre ellos.

\section{Categorías subásicas}

De igual manera se ha realizado un ANOVA $3 \times 13$ para analizar la utilización de los diferentes criterios explícitos generados por los sujetos en las categorías subásicas, no se han obtenido efectos principales de la variable Grupo $(\mathrm{F}=0.657 ; \mathrm{g} .1 .=2 ; \mathrm{p}=0.5223)$, ni efecto de interacción $(\mathrm{F}=1.328 ; \mathrm{g} .1 .=24$; $\mathrm{p}=0.1359$ ). Sin embargo, existe efecto principal de la variable criterios utiliza$\operatorname{dos}(F=19.32 ;$ g.l. $=12 ; p=1.0 E-4)$. Hemos aplicado la prueba de Tukey para saber entre qué niveles de la variable criterios se establecen las diferencias (DMS $=0,085)$. En la Tabla III podemos observar las medias obtenidas en cada grupo en la utilización de los diferentes criterios en las categorías subásicas.

\section{TABLA III}

Proporciones de utilización de los criterios para agrupar las enfermedades en categorias subásicas dentro de cada grupo

\begin{tabular}{lccccccccccccc}
\hline & C1 & C2 & C3 & C4 & C5 & C6 & C7 & C8 & C9 & C10 & C11 & C12 & C13 \\
\hline PROFANOS & .35 & .15 & .06 & .05 & .06 & .01 & .08 & 0 & .07 & .03 & .03 & .04 & .02 \\
\hline ENFERMERAS & .21 & .11 & .1 & .09 & .1 & .00 & .03 & .02 & .04 & .02 & .15 & .06 & .03 \\
\hline MEDICOS & .36 & .22 & .06 & .07 & .03 & .01 & .04 & 0 & .04 & .03 & .1 & .03 & .00 \\
\hline Totales & .31 & .16 & .07 & .07 & .07 & .00 & .05 & .00 & .05 & .03 & .09 & .04 & .02 \\
\hline
\end{tabular}

Existen diferencias entre el criterio localización y las demás. También existen diferencias entre causa y todas las demás excepto en relación específica. Entre todos los demás criterios no se han encontrado diferencias significativas. Por tanto, los tres grupos se comportan de manera similar, es decir, utilizan el mismo tipo de criterios explícitos para agrupar las enfermedades en las categorías subásicas. De igual manera, no hay una utilización diferente de los criterios en conjunto. El criterio de localización sigue siendo el más utilizado seguido por el criterio causa y relación específicas.

\section{Suprabásicas}

También se realizó un ANOVA $3 \times 13$ en las categorías suprabásicas. No se encontraron efectos principales de la variable Grupo $(\mathrm{F}=0.239$; g.l. $=2$; $\mathrm{p}=0.7884)$ ni con respecto a la interacción $(\mathrm{F}=0.918) ; \mathrm{g} .1 \mathrm{l}=24 ; \mathrm{p}=0.5774)$. Como en el caso anterior, sí existe efecto principal de la variable tipo de criterio utilizado en la clasificación ( $F=3.374 ;$ g.1. $=12 ; p=1 . \mathrm{OE}-4)$. En la Tabla IV se representan las medias de proporciones de utilización de los diferentes criterios utilizados en los agrupamientos de las categorías suprabásicas. Los criterios 
más ampliamente utilizados han sido el de localización y el de causa, no existiendo diferencias significactivas entre ellos.

\section{TABLA IV}

Proporciones de utilización de los criterios para agrupar las enfermedades en las categorías suprabásicas dentro de cada grupo

\begin{tabular}{lccccccccccccc}
\hline & C1 & C2 & C3 & C4 & C5 & C6 & C7 & C8 & C9 & C10 & C11 & C12 & C13 \\
\hline PROFANOS & .18 & .22 & .05 & 0 & .05 & 0 & .02 & .01 & .01 & 0 & .05 & 0 & .08 \\
\hline ENFERMERAS & .12 & .15 & .14 & .11 & .05 & .01 & 0 & 0 & 1 & 0 & 1 & 0 & .03 \\
\hline MEDICOS & .12 & .05 & .08 & .13 & .11 & 0 & .02 & 0 & .01 & .05 & .1 & 0 & 0 \\
\hline Totales & .14 & .14 & .09 & .1 & .05 & .02 & .01 & .00 & .01 & .01 & .09 & 0 & .02 \\
\hline
\end{tabular}

\section{Reorganización}

Se realizó un ANOVA $3 \times 13$ con medidas repetidas para analizar la utilización de los diferentes criterios en la tarea de reorganización para cada grupo. En la Tabla $\mathrm{V}$ podemos observar la proporción de utilización de los criterios para agrupar las enfermedades en la tarea de reorganización dentro de cada grupo. Los resultados muestran que no existe efecto principal de la variable Grupo $(\mathrm{F}=1,368 ; \mathrm{g} .1 .=2 ; \mathrm{p}=0.2629)$. Existe efecto principal de la variable tipo de criterio utilizado $(\mathrm{F}=12.662 ; \mathrm{g} .1 .=12 ; \mathrm{p}=1 . \mathrm{OE}-4)$. También existe efecto de interacción entre la variable Grupo y la variable tipo de categorías $(F=1.589$; g.l. $=24 ; \mathrm{p}=0.0371$ ).

\section{TABLA V}

Proporciones de utilización de los criterios para agrupar las enfermedades en la tarea de reorganización dentro de cada grupo

\begin{tabular}{lccccccccccccc}
\hline & C1 & C2 & C3 & C4 & C5 & C6 & C7 & C8 & C9 & C10 & C11 & C12 & C13 \\
\hline PROFANOS & .30 & .11 & .22 & .05 & .00 & 0 & .02 & .01 & .01 & .01 & .02 & .15 & .02 \\
\hline ENFERMERAS & .19 & .08 & .27 & .07 & 0 & .00 & .05 & 0 & .00 & .02 & .08 & .03 & 0 \\
\hline MEDICOS & .45 & .033 & .12 & .2 & .02 & .00 & .01 & 0 & .06 & .00 & .04 & .01 & 0 \\
\hline
\end{tabular}

Analizando si existen diferencias significativas, mediante la prueba de $\mathrm{Tu}$ key (DMS $=0,244$ ) dentro de cada categoría en los tres grupos encontramos que la única diferencia que se establece está en el criterio localización (C1). Existen diferencias entre la puntuación de las enfermeras y la de los médicos en el criterio localización. No existen diferencias entre los profanos y enfermeras y entre profanos y médicos. En los demás criterios no existen diferencias entre los grupos.

Si consideramos la diferente utilización de los criterios dentro de cada grupo, detectamos que en el grupo de profanos no existen diferencias entre el criterio localización (C1) y causa (C2), consecuencias (C3), tratamiento (C4) y mezcla de criterios (C12). Tampoco existen diferencias significativas entre tratamiento (C4), etiquetaje (C5), frecuencia (C6), tipo de personas o edades afectadas (C7), tipo de países (C8), sintomas (C9), duración (C10), relación especifica (C11) y otros (C13). Existen diferencias significativas entre el primer y segundo grupo de cri- 
terios. En el grupo de enfermería sólo existen diferencias entre el criterio consecuencias (C3) y el criterio etiquetaje (C5), frecuencia (C6), tipo de países (C8), sintomas (C9), duración (C10) y otros (C13). El criterio más utilizado por este grupo ha sido el de consecuencias, seguido por el de localización. En el grupo de los médicos únicamente existen diferencias significativas entre el criterio localización $(\mathrm{C} 1)$ y todos los demás, siendo el primero el más utilizado.

La utilización de los diferentes criterios es la misma en la primera clasificación que en la reorganización. Con respecto a las enfermeras sólo se aprecian diferencias significativas entre la utilización del criterio consecuencias en la tarea de reorganización y primera clasificación. Hay un aumento de la utilización de este criterio en la tarea de reorganización. En el grupo de médicos, el tipo de criterios utilizados para agrupar las enfermedades no cambia aun cuando se pide a los sujetos que reorganicen las tarjetas de otra manera. Con el análisis de conglomerados comprobaremos si realmente han vuelto a reorganizar las enfermedades utilizando los mismos criterios o si han utilizado los mismos agrupamientos que al principio.

\section{Análisis del contenido de los agrupamientos}

Para analizar el contenido de los agrupamientos que los sujetos realizaban en cada parte de la tarea hemos utilizado la técnica multivariada denominada análisis de conglomerados (análisis de clusters) (Arredondo, 1986). Mediante esta técnica podemos lograr comparar de forma cualitativa la clasificación de las enfermedades generadas por cada grupo y para cada aspecto de la tarea. $\mathrm{Pa}$ ra ello hemos construido matrices de similitud de las enfermedades utilizadas. La puntuación de similitud entre dos enfermedades las hemos operativizado como el número de sujetos, dentro de cada grupo, que habían situado dos enfermedades en el mismo montón. Disponemos de tres matrices, cada una correspondiente a los tres grupos (profanos, enfermeras y médicos), para las categorías básicas de la primera clasificación, otras tres matrices para las categorías generadas en la reorganización y otras tres para el recuerdo. Hemos analizado estas matrices mediante el programa HICLUS (Hierarchical clustering) de la serie MDS (X) desarrollado en la Universidad de Edimburgo, mediante el método «mínimo» (connectednes o single-link). Este método define la similaridad entre un punto y un conglomerado como la mayor similitud entre el punto externo y los puntos que constituyen el conglomerado. Utilizamos indistintamente los términos conglomerado y agrupamiento.

Los resultados de los análisis de conglomerados podemos observarlos en la representación construida en forma de diagramas de árbol. En la Figura 1 podemos encontrar los diagramas correspondientes a la primera clasificación de las categorías básicas, en los tres grupos. En la representación gráfica de los resultados obtenidos en el análisis de conglomerados podemos observar que en los niveles iniciales (en la parte izquierda) cada enfermedad forma un conglomerado o grupo, mientras que en los niveles más altos (parte derecha) todas las enfermedades forman un único grupo. Es decir, al nivel más bajo, cada enfermedad se considera como un grupo separado. En el nivel siguiente, los dos objetos más similares forman un nuevo grupo.

Primera clasificación (categorias básicas). Podemos observar que existen un número de agrupamientos similares en los tres grupos. Las enfermedades que se encuentran en los niveles iniciales y que por tanto tienen una similitud ma- 
yor son casi las mismas en los tres grupos (infarto de miocardio y angina de pecho; urticaria y acné; anginas, catarro y gripe). En términos generales vemos que existen agrupamientos comunes como los que integran las enfermedades del sistema nervioso (jaqueca y epilepsia), del sistema óseo (artritis, fractura de cadera y gota), enfermedades cardiovasculares (hipertensión, infarto de miocardio y angina de pecho) y enfermedades que afectan o su manifestación se produce en la piel (urticaria, acné y varicela). También presentan algunas diferencias, los médicos y los profanos agrupan los cánceres (cáncer de pulmón y cáncer de estómago) con algunas enfermedades como la úlcera y las enfermedades incluso con todas las demás enfermedades del sistema digestivo. Parece que aunque fundamentalmente la asociación se establece entre los dos tipos de cánceres, hay un número de personas en los tres grupos que agrupa estas enfermedades por ęl sistema corporal afectado. De ahí que el cáncer aparezca agrupado con las enfermedades del aparato digestivo.

Donde observamos las principales diferencias es en la aparición de un conglomerado muy numeroso en el grupo de los médicos, donde se incluyen las enfermedades infecciosas (sida, hepatitis, malaria, polio, neumonía, varicela, gripe, anginas y catarro). En el grupo de enfermeras también se observa este conglomerado de enfermedades infecciosas aunque incluyen en él un menor número de enfermedades (malaria, polio, neumonía, anginas, gripe y catarro). En los profanos no se aprecia este agrupamiento. En vez de eso, agrupan parte de las enfermedades infecciosas en un agrupamiento de enfermedades comunes respiratorias (neumonía, gripe, anginas y catarro). Las demás enfermedades que no se unen entre sí en los niveles iniciales o intermedios no guardan una gran similitud con los conglomerados ya existentes.

Reorganización. Los grupos de enfermedades más semejantes entre sí y que, por tanto, se sitúan en los niveles iniciales siguen siendo las mismas para los tres grupos (infarto de miocardio y angina de pecho; gripe y catarro). También siguen apareciendo agrupamientos comunes a los tres grupos, como el que hace referencia al sistema óseo. En los grupos de los profanos y enfermeras aparecen dos agrupamientos que incluyen mayor número de enfermedades. Estas las podemos denominar enfermedades graves: sida, infarto de miocardio, angina de pecho, cáncer de pulmón, y cáncer de estómago, en el grupo de enfermeras, y sida, hipertensión, infarto de miocardio, angina de pecho, cáncer de pulmón y cáncer de estómago, en el grupo de profanos. De la misma manera aparece otro conglomerado que hemos denominado enfermedades comunes o leves: neumonía, anginas, gripe, catarro y miopía en el grupo de los profanos y más amplio que incluyen un mayor número de enfermedades. Es decir, las mismas que el grupo anterior junto con urticaria y acné en el grupo de las enfermeras, ya que en el caso de los profanos está separada. A este respecto, también en el grupo de los médicos surge un conglomerado con enfermedades leves muy similar al de las enfermeras (neumonía, anginas, gripe, catarro, más varicela, urticaria y acné). Sin embargo, este grupo sigue diferenciando entre enfermedades cardiovasculares y factores de riesgo (hipertensión, infarto de miocardio, angina de pecho, diabetes) y otro grupo que incluye, además de los cánceres, algunas enfermedades del aparato digestivo.

Existen algunos agrupamientos que no son compartidos por los tres grupos. En el grupo de los profanos podemos observar que diabetes y anemia, enfermedades que tienen relación con la sangre, están unidas en un agrupamiento y apendicitis y úlcera como enfermedades del aparato digestivo. Sin embargo, este último 


\section{6}

FIGURA 1

Representación gráfica del análisis de conglomerados de la primera clasificación (categorías básicas)

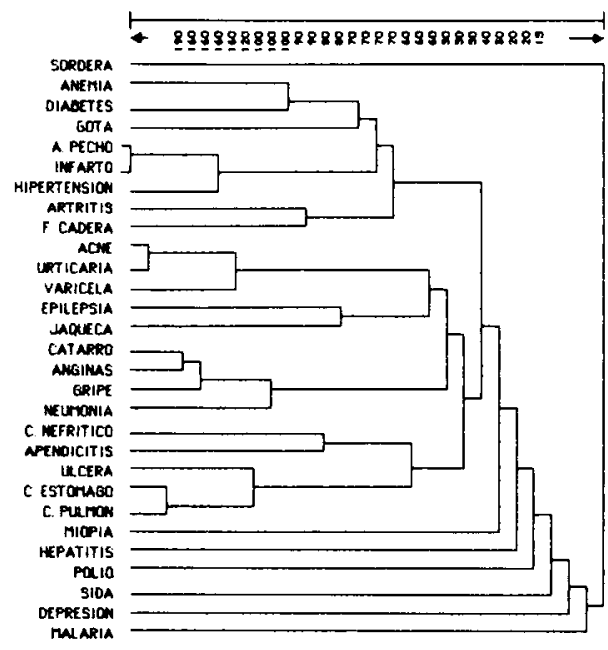

PROFANOS

ENFERMERAS
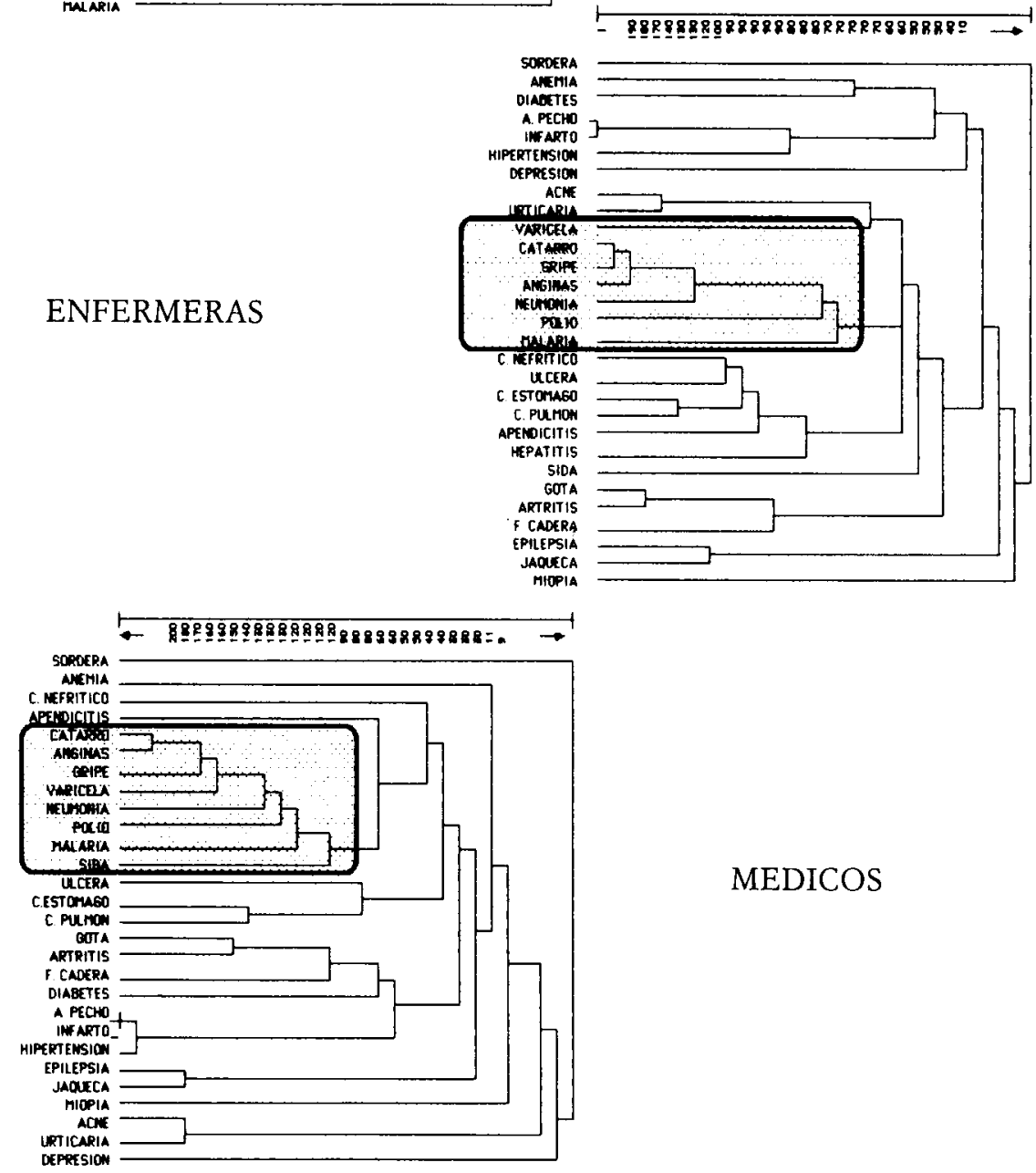
sí aparece en las enfermeras. En los médicos aparecen las enfermedades neurológicas (jaqueca y epilepsia) y la malaria y anemia de difícil definición, ya que pueden ser o bien porque son enfermedades hematológicas o bien porque la malaria puede producir anemia. Además hay que señalar que este último agrupamiento presenta una asociación en niveles muy altos, con lo cual su similitud no es tan grande como si apareciera en los niveles iniciales.

Recuerdo de la primera clasificación (categorias básicas). Cuando comparamos en los tres grupos el diagrama de árbol obtenido en la primera clasificación en los tres grupos con el diagrama de árbol en la fase de recuerdo, podemos observar que los médicos son los que presentan los agrupamientos más similares. Las variaciones son mínimas e incluso los conglomerados más próximos situados en los niveles iniciales son los mismos. En el grupo de enfermeras las diferencias son mayores. En vez de recordar el grupo de las víricas, han agrupado en la fase de recuerdo las enfermedades víricas del aparato respiratorio únicamente. Mientras que en la primera clasificación ponían juntas los cánceres y las digestivas, en la tarea de recuerdo las separan en dos conglomerados diferentes. En el grupo de profanos también aparecen pequeñas modificaciones. Incluso hay un agrupamiento formado en la primera clasificación (diabetes y epilepsia) que no aparece en la tarea de recuerdo. Fundamentalmente, con pequeñas variaciones, los agrupamientos realizados por cada grupo se mantienen, lo que cambia es la inclusión de una $\mathrm{u}$ otra enfermedad.

\section{DISCUSION}

Nuestro objetivo principal era analizar qué tipo de dimensiones o criterios utilizan los profanos, enfermeras y médicos cuando clasifican enfermedades. Con respecto a los criterios explícitos utilizados por los sujetos para agrupar las enfermedades en la primera clasificación (categorías básicas), podemos observar que el criterio más utilizado es el de localización anatómica. No hay diferencias significativas entre este criterio y los demás. La diferencia entre los tres grupos radica en que los médicos utilizan este criterio en mayor medida que los profanos y las enfermeras. Por tanto, podemos concluir que en esta tarea no hay una utilización diferencial de los criterios según el grupo de sujetos, aunque los médicos utilicen este criterio en mayor medida que los demás grupos. Cuando analizamos el contenido de los agrupamientos llegamos a la conclusión, que los profanos se diferencian de los médicos y de las enfermeras fundamentalmente porque estos últimos utilizan agrupamientos de enfermedades infecciosas, mientras que no aparece este tipo de agrupamiento en los profanos. A la vista de los resultados parece que este tipo de agrupamiento es característico de los dos últimos grupos debido a que es una de las características de la clasificación médica. Con respecto a esta primera clasificación podemos concluir que los profanos comparten en cierta medida el modelo médico ya que muchos de los agrupamientos los han realizado por localización anatómica de las enfermedades. Sin embargo, no utilizan uno de los criterios empleados desde el punto de vista de la clasificación médica como es el caso de las enfermedades infecciosas. Lalljee, Lamb y Carnibella (en prensa) también encontraron, utilizando la misma tarea, que este criterio resultaba muy relevante en el agrupamiento de enfermedades aunque en nuestro estudio ha resultado ser más utilizado que en el de los anteriores autores citados. 
Nuestros datos no coinciden con los encontrados por Bishop (1991b). La dimensión contagio no parece ser tan relevante, ya que únicamente los médicos y las enfermeras la han utilizado en sus agrupamientos. Sin embargo, con respecto a la reorganización de la clasificación encontramos que todos los grupos utilizan el criterio gravedad. Este resultado sí apoya la segunda dimensión detectada por Bishop (1991b). Pensamos que estas diferencias son debidas a la metodología utilizada. Este autor empleó el análisis multidimensional, e imponía ciertas restricciones al número de agrupamientos que los sujetos podían realizar, que en este caso era de 10 . Nosotros no sometimos a los sujetos a ningún tipo de restricción, ni les suministrábamos ninguna escala en la que tuvieran que valorar cada enfermedad en relación a aspectos tales como contagio, gravedad, etc. Además queremos señalar que al ser la muestra de profanos utilizada de personas alrededor de 30 años que poseían una formación universitaria, este factor ha podido influir en los resultados. Por ejemplo, Helman (1978) también encontró modelos más cercanos al modelo médico en sujetos de generaciones jóvenes comparados con los mayores. ¿Qué sucedería si utilizásemos sujetos de más edad y sin estudios universitarios? ¿Qué tipo de criterios utilizarían? Probablemente encontraríamos mayores diferencias con los profesionales de la salud. Y si utilizáramos diferentes enfermedades, ¿seguiríamos encontrando los mismos resultados?

Las medidas utilizadas para poder conocer la clasificación jerárquica no han discriminado entre los tres grupos. No hemos encontrado diferencias en las medidas cuantitativas de la clasificación jerárquica (MCBSP, MCSBB, número de categorías básicas y número de categorías suprabásicas). La única diferencia que hemos encontrado ha sido en el número de categorías subásicas. Los médicos realizan un mayor número de subdivisiones que las enfermeras y éstas, a su vez, mayor número que los profanos. Aquí podemos ver la influencia del modelo médico que permite establecer una mayor diferenciación entre las enfermedades y que probablemente proporciona a los médicos una mayor diferenciación conceptual. Los mismos resultados se han encontrado en los criterios explícitos utilizados por los diferentes grupos. No hemos encontrado diferencias en los criterios de la clasificación subásica ni en los empleados en las categorías suprabásicas. Las clasificaciones jerárquicas son muy similares en los tres grupos. Sin embargo, el tipo de criterios incluidos en las categorías suprabásicas no era diferente de los incluidos en las demás categorías. Es decir, no ha habido una clasificación jerárquica de conceptos en ninguno de los tres grupos. Los conceptos suprabásicos no son de mayor nivel de abstracción que los demás. Y esto sucede también con las clasificaciones realizadas por los médicos. El intento de comparar los resultados obtenidos en el área de la Física con los de la Medicina no se ha coronado con éxito. Quizá la razón de no haber encontrado una clasificación realmente jerárquica haya sido el tipo de material empleado. Pensemos que Chi, Feltovich y Glaser (1981) utilizaron problemas de Física, lo cual no es totalmente idéntico, desde el punto de vista conceptual, a las enfermedades. En ese caso podríamos haber utilizado como material descripciones de personas con un conjunto de síntomas. Creemos que sería interesante utilizar en próximas investigaciones este tipo de material.

En la tarea de reorganización los profanos y las enfermeras disminuyeron el número de agrupamientos realizados, con lo que en un número menor de grupos incluyeron mayor número de enfermedades. No ocurre así con los médicos. Generan el mismo número de agrupamientos que en la primera clasificación 
(categorías básicas). Con respecto a los criterios explícitos utilizados, los médicos utilizan en mayor medida el criterio de localización anatómica para agrupar las enfermedades que los profanos y las enfermeras muestran una utilización intermedia de este criterio. No se aprecian diferencias entre los demás criterios. Si comparamos los criterios de la primera clasificación y la reorganización, podemos observar que no existen diferencias en el grupo de profanos y médicos, pero sí en el grupo de enfermería, donde en la tarea de reorganización parecen recurrir más al criterio de consecuencias para agrupar las enfermedades que en la primera clasificación. Una posible explicación de este resultado es que éste cumple una función muy importante en la actividad profesional del personal de enfermería.

Cuando analizamos el contenido de los agrupamientos realizados por los grupos de profanos y enfermería detectamos que los conglomerados son mayores. Es decir, incluyen mayor número de enfermedades que en la primera clasificación y utilizan fundamentalmente conglomerados de enfermedades graves y leves. Esto apoya los resultados encontrados en la utilización de los criterios explícitos por parte de las enfermeras. En el caso de los profanos, aunque hay un aumento de la utilización de este criterio en la tarea de reorganización, no muestra diferencias significativas con el de localización. La reorganización no es total. Muchos conglomerados, sobre todo los que se basan en agrupamientos según según la localización anatómica, siguen siendo los mismos. Se agrupan en conglomerados mayores en esta tarea. Los médicos, sin embargo, han incluido un agrupamiento de enfermedades leves o comunes, pero no han agrupado las enfermedades graves.

Respecto al último objetivo, se confirma la hipótesis de que los médicos tienen un mejor recuerdo de sus primeras clasificaciones (categorías básicas) que los profanos y las enfermeras. Estos dos grupos se comportan de manera semejante respecto a este criterio. Se ha observado esta confirmación tanto en el análisis cuantitativo como en el análisis de los conglomerados.

Los profanos comparten en gran medida el mapa clasificatorio de las enfermedades con los médicos y enfermeras. Pero lo más interesante es ¿qué tipo de aspecto es el que comparten de dicha clasificación? La respuesta puede ser el aspecto que les resulta más útil. La organización del sistema sanitario está dividido en especialidades basadas en la localización anatómica del trastorno. Así se dispone de oftalmólogo, de especialista del aparato digestivo, de pulmón y corazón, etc. De tal manera que cuando percibimos un cambio en nuestro funcionamiento corporal, lo primero que hacemos es situar dónde se encuentra. Si nos duele el estómago lo más adecuado es dirigirnos al médico de cabecera para solicitarle un volante para el especialista de digestivo. Por tanto, clasificar las enfermedades según su localización anatómica implica conocer a dónde acudir ante un síntoma de alarma. Sin embargo, la cualidad de infecciosas de las enfermedades no es un criterio tan pragmático como el anteriormente mencionado.

Parece que el sistema médico ha impregnado los modelos de los profanos cuando es útil para la acción contra la enfermedad. Esto aporta resultados confirmatorios a la afirmación de Fitzpatrick (1984) que señala que no se debe sobreestimar la diferencia entre los modelos profanos y los médicos. Desde luego en el aspecto del dominio de clasificación de enfermedades no hemos encontrado ese «abismo» entre los dos modelos. Al mismo tiempo ilustra una de las características de las teorías intuitivas: el criterio pragmático que rige el pensamiento intuitivo (Carretero y García Madruga, 1984; Claxton, 1984). 


\section{Referencias}

Arredondo, J. M. (1986). Análisis de conglomerados y su aplicación en la Psicología Ambiental. F. Jiménez Burillo, J. I. Aragonés (Comps.) Introducción a la Psicología Ambiental. Madrid: Alianza Editorial.

Baumann, L. J.; Cameron, L. D.; Zimmerman, R. S., y Leventhal, H. (1989). Illness representations and matching labels with symptoms. Health Psychology, 8 (4), 449-469.

BIsнop, G. D. (1991a). Understanding the undertanding of illness: lay disease representations. En: J. A. Skelton y R. T. Croyle (Eds.). Mental representation in bealth and illness. SpringerVerlag.

Bishop, G. D. (1991b). Lay diesease representations and responses to victims of disease. Basic and Applied Social Psychology, 12 (1), 115-132.

Bishop, G. D.; Briede, C.; Cavazos, L.; Grotzinger, R., y McMahon, S. (1987). Processing illness information: the role of disease prototypes. Basic and Applied Social Psychology, 8 (1-2), 21-45.

Carretero, M., y García Madruga. J. A. (1984). (Comps.). Lecturas de Psicología del Pensamiento. Madrid: Alianza Editorial.

Claxton, G. (1984). Live and Learn. Londres: Harper \& Row. Trad. cast. de C. González: Vivir $y$ aprender. Madrid: Alianza, 1987.

Chi, M. T. H.; y Feltovich, P. J., y Glaser, R. (1981). Categorization and representation of physics problems by experts and novice. Cognitive Science, 5, 121-151.

ChI. M. T. H.; Glaser, R., y Rees, R. (1982). Expertise in problem solving. En R. Sternberg (Ed.): Advances in the Psychology of Human intelligence. Vol. 2. Hillsdale, N.J.: Erlbaum.

ChI, M. T. H.; Glaser, R., y Farr, M. J. (1988). The nature of expertise. Hillsdale, N.J.: Erlbaum.

Dandrade, R. G.; Quinn, N.; Nerlove, S. B., y Romney, A. K. (1972). Categories of Disease in American English and Mexican-Spanish. En A. K. Romney, R. G. Shepard y S. B. Nerlove (1972). Multidimensional Scaling Seminar Press.

Fitzpatrick, R. (1984). Lay concepts of illness. En R. Fitzpatrick, J. Hinton, S. Newman, G. Scambler y J. Thompson (Eds.). The experience of illness. Tavistock.

Helman, C. (1978). Feed a cold, starve a fever: folk models of infection in an English suburban community and their relation to medical treatment. Culture, Medicine and Psychiatry, 2, 107-137.

Jenkins, C. D. (1966). Group differences in perception: A study of community beliefs and feeling about tuberculosis. American Joumal of Sociology, 71, 417-426.

Jennkins, C. D., y Zyzanski, S. J. (1968). Dimensions of belief and felling concerning three diseases, poliomyelitis, cancer, and mental illness: A factor analytic studyc. Behavioral Science, 13, 372-381.

Lalljee, M.; Lamb, R., y Carnibella, G. (en prensa). Lay prototypes of illness: their content and use.

Lau, R. R., y Hartman, K. A. (1983). Common sense representation of common illnesses. Health Psycbology, 2, 167-185.

LaU, R. R.; Bernard, I. M., y Hartman, K.A. (1989). Further explorations of common-sense representations of common illness. Health Psychology, 8 (2), 195-219.

Leventhal, H.; Meyer, D., y Nerenz, D. (1980). The common-sense representation of illness danger. En S. Rachman (Eds.). Contributions to medical Psychology. Vol II.

Leventhal, H., y Nerenz, D. R. (1982). A model for stress research and some implications for the control of stress disorders. En D. Meichembaum y M. Jarremko (Eds.). Stress prevention and management: a cognitive bebavioral approach Nueva York: Plenum Press.

LÓPEZ MANjón, A. (1991). «Teorias intuitivas sobre la enfermedad: categorización, representación y razonamiento en expertos y profanos». Tesis doctoral inédita. Dpt. de Psicología Básica, Social y Metodología. Universidad Autónoma de Madrid.

San Martín, R., y Pardo, A. (1989). Psicoestadística. Contrastes paramétricos y no paramétricos. Madrid: Pirámide.

Turk, D. C.; Rudy, T. E., y SAlovey, P. (1986). Implicit models of illness. Journal of Bebavioral Medicine, 9 (5), 453-474. 